\title{
Biomarkers in the accurate subclassification of non-small-cell lung carcinoma for targeted therapy: issues and prospects
}

First draft submitted: 18 February 2017; Accepted for publication: 1 March 2017; Published online: 16 June 2017

Keywords: immunohistochemical stains • lung cancer classification • non-small-cell lung carcinoma $\bullet$ protein biomarkers

Lung cancer is still among the most common causes of cancer-related death in the USA and worldwide [1]. Lung cancers are a heterogeneous group of tumors which consist of many histomorphological subtypes $[2,3]$. Until the past decade, 'non-small-cell lung carcinoma (NSCLC)' and 'small-cell lung carcinoma' were the most frequently used diagnostic terms for lung cancer in oncological practice. Due to the limited treatment options that were available, there was no need for further subclassification of lung carcinomas. However, there have been tremendous advances in recent years. Advanced molecular and genetic analyses have identified different driver gene mutations and intracellular signaling pathways in different subtypes of NSCLC. The most well-studied subtypes are adenocarcinoma (ADC) and squamous cell carcinoma $(\mathrm{SqCC})[4,5]$. These two subtypes of NSCLC account for approximately $70-80 \%$ of lung cancers. In ADC, the discovery of $E G F R$ mutations and $A L K$ rearrangements lead to the development of targeted therapies for lung cancer patients whose tumors harbor these molecular abnormalities, such as, EGFR tyrosine kinase inhibitors for EGFR mutations and ALK inhibitors for ALK rearrangements [5]. Other examples include pemetrexed which is effective only in patients with advanced ADCs, nivolumab (a programmed death-ligand antibody) which is effective only in patients with advanced SqCCs and bevacizumab which is contraindicated in patients with SqCC due to the complication of pulmonary hemorrhage [2-5]. Taken together, the clinical application of targeted therapy is dependent on the accurate subclassification of the tumor.

\section{Current guidelines in the subclassification of lung cancer}

Clinically, the majority of lung cancer patients present with locally advanced disease or with distant metastasis at the time of diagnosis, in which case, surgical resection for morphological evaluation and subclassification of the tumor may not be an option. Thus, transthoracic and/or transbronchial fine needle aspiration biopsy of the tumor become important approaches to obtain tumor tissue for diagnosis, staging and subclassification as well as for molecular characterization [6]. Due to the heterogeneity of lung cancer, accurate diagnosis on small biopsies can be challenging $[2,3]$. In addition to histomorphological evaluation, immunohistochemical (IHC) biomarkers play a critical role in the accurate subclassification of lung tumors.

Based on current molecular knowledge sification of lung cancer, the new edition of WHO classification of lung tumors and the and the clinical demand for accurate subclas-

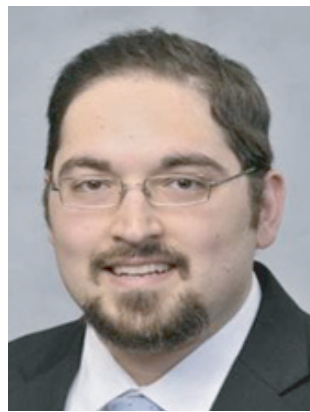

Lais Osmani

Department of Pathology, The Johns Hopkins Medical Institutions, Baltimore, Maryland 21224, USA

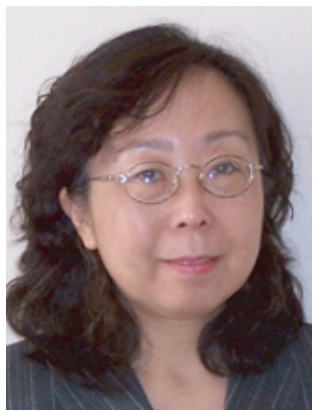

Qing Kay Li Department of Pathology, The Johns Hopkins Medical Institutions, Baltimore, Maryland 21224, USA Author for correspondence: qli23@jhmi.edu 
International Association for the Study of Lung Cancer recommend updated guidelines for diagnosing lung cancer, which were published in 2015 [2,3]. The new guidelines emphasize: the utility of IHC markers in the subclassification of lung cancer, the critical role of molecular characterization of tumors, unique terminology for the subclassification of NSCLC using small biopsy specimens, a completely different approach to the subclassification of ADC, the subclassification of $\mathrm{SqCC}$ into keratinizing, nonkeratinizing and basaloid subtypes as well as several other recommendations. These new guidelines will have significant impact on daily practice and patient care. They emphasize the critical role of biomarkers in the accurate subclassification of lung cancer.

\section{Limitations of current IHC markers}

In daily clinical practice, the most commonly used IHC biomarkers are TTF-1, napsin A, mucin, CK5/6, $\mathrm{P} 63$ and P40 for the classification of NSLCLs $[7,8]$. In NSCLC, TTF-1 and napsin A are expressed by pneumocytes and are most useful for the identification of ADCs, whereas both P63 and P40 (a truncated form of P63) are expressed by the basal or progenitor cell layer of bronchial epithelium and are useful for the identification of SqCCs. CK5/6 is also used for the identification of SqCC. These biomarkers have different sensitivities and specificities. Our studies and others' studies have demonstrated that these markers have sensitivities and specificities ranging from approximately $70-100 \%$ for the identification of ADCs, and from approximately $60-100 \%$ for the identification of SqCCs [7,8]. Limitations of these biomarkers include: the requirement of multiple tissue sections for IHC study, which may exhaust tumor tissue, compromising the molecular characterization of the tumor in small biopsy samples; the time-consuming nature of IHC testing, which can result in prolonged turnaround times, significantly affecting the patient's clinical management; the interpretation of the IHC results may be influenced by nonspecific staining of non-neoplastic cells, such as, pulmonary alveolar macrophages and bronchial basal cells; and different clones of the same antibody may have different effects on the IHC staining pattern.

To improve the accuracy of the subclassification of NSCLC, we have proposed combining individual markers (TTF-1, napsin A and P40) into a novel triple marker. In our study, the triple marker demonstrates a similar sensitivity and specificity as the individual IHC biomarkers. It has demonstrated a sensitivity of $86.0 \%$ and a specificity of $100 \%$ in lung ADCs, and a sensitivity of $100 \%$ and a specificity of $97.1 \%$ in lung SqCCs $[9,10]$. Our practice also indicates that the util- ity of a combined IHC approach is a cost-effective method of subclassifying lung cancer in daily practice. Furthermore, the triple marker improves turnaround time and has the advantage of using minimal tumor tissue. However, the nonspecific staining of normal pulmonary cells, such as, alveolar macrophages and bronchial basal cells, is still a limitation of the triple marker. Therefore, alternative approaches of using IHC biomarkers and/or the discovery of new biomarkers are necessary to fulfill clinical demands and the rising standard of care, which includes targeted therapy and personalized medicine.

\section{Prospective}

Lung cancer development and progression are multistep processes. They are characterized by aberrant genetic and protein expression, which subsequently lead to phenotypic transformation of cells and progression of the tumor [2-5]. This process involves complex intracellular signaling pathways and various cellular proteins [11-13]. In addition to the genetic characterization of lung cancer, it is also necessary to address the need for protein biomarkers for the accurate subclassification of lung cancer as well as for monitoring disease progression and treatment response [14]. Based on advanced molecular technologies and proteomics, numerous studies have reported candidate biomarkers in lung cancer. These state-of-the-art technologies provide new platforms for the systematic study of intracellular proteins and for the characterization of the complex alveolar microenvironment. The keyword search query of 'protein biomarkers and lung cancer' in PubMed yields more than 20,000 articles. This large amount of data represents the general efforts of many in the discovery of potential biomarkers for lung cancer. However, none of these biomarkers are widely used in daily practice. Recently, SPATS2 has been proposed for the identification of SqCC and ST6GALNAC1 for the identification of ADC [15]. The sensitivities and specificities of these candidate biomarkers still need to be validated in well-designed, large-scale studies.

\section{Conclusion}

Targeted therapies for lung cancer have progressed rapidly; however, the overall progression-free survival rate of lung cancer patients is still suboptimal. In order to improve clinical outcomes for lung cancer patients, biomarkers for the accurate subclassification of tumors as well as for monitoring response to targeted therapy, are needed. Recent advances in molecular techniques and proteomics allow for systematic methods to discover and evaluate thousands of biomarkers in a single experimental setting. Although select candidate biomarkers have been studied and evaluated using clinical 
specimens, further improvement of the workflow and validation process in large scale cohorts is still needed.

\section{Financial \& competing interests disclosure}

The authors have no relevant affiliations or financial involvement with any organization or entity with a financial inter-

\section{References}

1 Siegel RL, Miller KD, Jemal A. Cancer Statistics, 2017. CA Cancer J. Clin. 67(1), 7-30 (2017).

2 Travis WD, Brambilla E, Burke AP, Marx A, Nicholson AG. WHO Classification of Tumours of the Lung, Pleura, Thymus and Heart. International Agency for Research on Cancer, Lyon, France (2015).

3 Travis WD, Brambilla E, Nicholson AG et al. The 2015 World Health Organization classification of lung tumors: impact of genetic, clinical and radiologic advances since the 2004 classification. J. Thorac Oncol. 10(9), 1243-1260 (2015).

4 Cancer Genome Atlas Research Network. Comprehensive genomic characterization of squamous cell lung cancers. Nature 489(7417), 519-525 (2012).

5 Cancer Genome Atlas Research Network. Comprehensive molecular profiling of lung adenocarcinoma. Nature 511(7511), 543-550 (2014).

6 Feller-Kopman D, Yung RCW, Burroughs F, Li QK. Cytology of endobronchial ultrasound-guided transbronchial needle aspiration: a retrospective study with histology correlation. Cancer Cytopathol. 117(6), 482-490 (2009).

7 Gurda GT, Zhang L, Wang Y et al. Utility of five commonly used immunohistochemical markers TTF-1, Napsin A, CK7, CK5/6 and P63 in primary and metastatic adenocarcinoma and squamous cell carcinoma of the lung: a retrospective study of 246 fine needle aspiration cases. Clin. Transl. Med. 4, 16 (2015).

8 Lilo MT, Allison D, Wang Y et al. Expression of P40 and $\mathrm{P} 63$ in lung cancers using fine needle aspiration cases. est in or financial conflict with the subject matter or materials discussed in the manuscript. This includes employment, consultancies, honoraria, stock ownership or options, expert testimony, grants or patents received or pending, or royalties.

No writing assistance was utilized in the production of this manuscript.

Understanding clinical pitfalls and limitations. J. Am. Soc. Cytopathol. 5(3), 123-132 (2016).

9 Ao $\mathrm{MH}$, Zhang $\mathrm{H}$, Sakowski L et al. The utility of a novel triple marker (combination of TTF1, napsin A and p40) in the subclassification of non-small cell lung carcinoma. Hum. Pathol. 45(5), 926-934 (2014).

10 Sharma R, Wang Y, Chen L et al. Utility of a novel triple marker (combination of thyroid transcription factor 1 , Napsin A and P40) in the subclassification of non-small cell lung carcinomas using fine needle aspiration cases. Hum. Pathol. 54, 8-16 (2016).

11 Zupa A, Improta G, Silvestri A et al. A pilot characterization of human lung NSCLC by protein pathway activation mapping. J. Thorac. Oncol. 7(12), 1755-1766 (2012).

12 Krencz I, Sebestyén A, Fábián K et al. Expression of mTORC1/2-related proteins in primary and brain metastatic lung adenocarcinoma. Hum. Pathol. doi:10.1016/j. humpath.2016.12.012 (2016) (Epub ahead of print).

13 Kadara H, Choi M, Zhang J et al. Whole-exome sequencing and immune profiling of early-stage lung adenocarcinoma with fully annotated clinical follow-up. Ann. Oncol. pii:mdw436 (2016) (Epub ahead of print).

14 Li QK, Gabrielson E, Zhang H. Application of glycoproteomics for the discovery of biomarkers in lung cancer. Proteomics Clin. Appl. 6(5-6), 244-256 (2012).

15 Takamochi K, Ohmiya H, Itoh M et al. Novel biomarkers that assist in accurate discrimination of squamous cell carcinoma from adenocarcinoma of the lung. BMC Cancer 16(1), 760 (2016). 